\title{
Correction to: Multimedia Cloud Computing Systems
}

\section{Correction to:}

\section{A. Salehi, X. Li,}

Multimedia Cloud Computing Systems

https://doi.org/10.1007/978-3-030-88451-2

The book was inadvertently published with an incorrect affiliation for one of the author Xiangbo Li as "Brightcove, Scottsdale AZ, USA". It has been updated as

"Twitch Interactive Inc., San Francisco, CA, USA" 